\title{
Recombinant Follicular Stimulating Hormone Plus Recombinant Luteinizing Hormone Versus Human Menopausal Gonadotropins- Does the Source of LH Bioactivity Affect Ovarian Stimulation Outcome?
}

\author{
Michal Kirshenbaum ( $\nabla$ michal.siegel@gmail.com ) \\ Sheba Medical Center at Tel Hashomer https://orcid.org/0000-0001-8441-7724 \\ Or Gil
}

Tel Aviv University Sackler Faculty of Medicine

Jigal Haas

Sheba Medical Center at Tel Hashomer: Sheba Medical Center

Ravit Nahum

Sheba Medical Center at Tel Hashomer: Sheba Medical Center

Eran Zilberberg

Sheba Medical Center at Tel Hashomer: Sheba Medical Center

Oshrit Lebovitz

Sheba Medical Center at Tel Hashomer: Sheba Medical Center

Raoul Orvieto

Sheba Medical Center at Tel Hashomer: Sheba Medical Center

\section{Research}

Keywords: Assisted reproductive technology, Gonadotropins, Human chorionic gonadotropin, Luteinizing hormone, Ovarian stimulation

Posted Date: August 6th, 2021

DOI: https://doi.org/10.21203/rs.3.rs-772132/v1

License: (c) (i) This work is licensed under a Creative Commons Attribution 4.0 International License.

Read Full License

Version of Record: A version of this preprint was published at Reproductive Biology and Endocrinology on December 1st, 2021. See the published version at https://doi.org/10.1186/s12958-021-00853-7. 


\section{Abstract}

Background: Luteinizing hormone (LH) and human chorionic gonadotropin (hCG) activate distinct intracellular signaling cascades. However, due to their similar structure and common receptor, they are used interchangeably during ovarian stimulation (OS). This study aims to assess if the source of LH used during OS affects IVF outcome.

Materials and methods: This was a cross sectional study of patients who underwent two consecutive IVF cycles, one included recombinant follicular stimulating hormone (FSH) plus recombinant LH [rFSH+rLH, (Pergoveris)] and the other included urinary hCG [highly purified hMG (HP-hMG), (Menopur)]. The OS protocol, except of the LH preparation, was identical in the two IVF cycles.

Results: The rate of mature oocytes was not different between the treatment cycles $(0.9$ in the rFSH+rLH vs 0.8 in the HP-hMG, $p=0.07)$. Nonetheless, the mean number of mature oocytes retrieved in the $\mathrm{rFSH}+\mathrm{rLH}$ treatment cycles was higher compared to the HP-hMG treatment cycles ( $10 \pm 5.8$ vs $8.3 \pm 4.6$, respectively, $\mathrm{P}=0.01$ ). Likewise, the mean number of fertilized oocytes was higher in the $\mathrm{FFSH}+\mathrm{rLH}$ cycles compared with the HP-hMG cycles $(8.5 \pm 5.9$ vs $6.4 \pm 3.6$, respectively, $p=0.05)$. There was no difference between the treatment cycles regarding the number of top-quality embryos, the ratio of top-quality embryos per number of oocytes retrieved or fertilized oocytes or the pregnancy rate.

Conclusion: The differences in treatment outcome, derived by different LH preparations reflect the distinct physiological role of these molecules. Our findings may assist in tailoring a specific GT regimen when assembling an OS protocol.

\section{Introduction:}

Ovarian stimulation (OS) is a fundamental step in the process of artificial reproductive technology (ART). Administration of exogenous gonadotropins enables the recruitment and development of multiple follicles, in order to yield an optimal number of mature oocytes. The action of both Follicular stimulating hormone (FSH) and luteinizing hormone (LH) is required for follicular growth (1). While FSH is the main regulator of follicular recruitment and genesis by stimulating granulosa cell proliferation and differentiation, the benefit of LH treatment may be derived from its effect on the synthesis of follicular steroids. Moreover, LH exerts an antiapoptotic effect on granulosa cells and promotes a paracrine signaling involved in cell expansion and oocyte maturation during folliculogenesis (2).

The first available source of LH was produced from the urine of postmenopausal women, known as human menopausal gonadotropin ( $\mathrm{hMG}$ ), which contained a mixture of $\mathrm{FSH}$, human chorionic gonadotropin ( $\mathrm{hCG}$ ) and LH in varying amounts (3). Improvement in purification techniques enabled the development of highly purified- $\mathrm{hMG}(\mathrm{HP}-\mathrm{hMG})$ containing 1:1 ratio of $\mathrm{FSH}$ and $\mathrm{LH}$ bioactivity that is predominantly derived from hCG, since LH molecules are lost during the purification process. Another source of commercial gonadotropin is recombinant gonadotropin preparations. These include recombinant FSH (rFSH), rLH, rhCG and combined product of $\mathrm{rFSH}$ and rLH in a 2:1 ratio (4). 
hCG and LH are structurally similar molecules, composed of two heterodimeric glycoprotein subunits, a mutual alfa subunit and a distinct beta subunit, which differs in length, post-translational glycosylation and structure (5). Considering LH and hCG similar structure, their common receptor (LH/CGR) and data suggesting that $\mathrm{hCG}$ has a role throughout the menstrual cycle, they are currently used interchangeably in ART protocols to drive OS. Nonetheless, in vitro studies revealed specific bioactivity effects of LH and hCG, which differ by triggering separate intracellular signaling cascades. LH binding to the LH/CG receptor results in a more potent activation of the proliferative and anti-apoptotic pathways, whereas hCG has a higher potency for activation of the steroidogenic pathways (5-7).

Although the physiologic role of LH during the follicular phase of natural cycle is unequivocal, the importance of exogenous preparations with LH bioactivity during OS in normo-gonadotrophic women is of great debate. Previous randomized controlled trials and meta-analysis have demonstrated the benefit of adding different LH bioactivity preparations to FSH during OS, compared to FSH treatment only. Treatment with HP-hMG resulted in higher proportion of top- quality embryos developed, compared with rFSH. Moreover, at the end of stimulation, higher estradiol and lower progesterone levels were measured in the HP-hMG cycles compared with the rFSH cycles. A trend towards higher ongoing pregnancy rate and live birth rate was observed in the fresh HP-hMG cycles versus rFSH alone (8-10). A systemic review assessing the role of rLH supplementation in OS in specific subgroups of patients demonstrated its benefit in women with normal ovarian reserve parameters and a hypo-response to OS and in women 3639 years of age (11). The superiority of HP-hMG or the combination of rLH with rFSH over rFSH alone during $O S$ is thought to be attributed to the LH activity, which induces differences in the synthesis of follicular steroids, impacts oocyte maturation and improves embryo quality and endometrial receptivity $(1,12,13)$.

In clinical practice, it is essential to consider all aspects when choosing the most appropriate gonadotropin therapy for OS. Since hCG and LH activate different physiological events, different commercially available LH preparation ( $\mathrm{hMG}$ and $\mathrm{rFSH}+\mathrm{rLH}$ ) may affect the stimulation characteristics and consequently impact clinical outcomes. Nonetheless, rLH and hMG are still considered equivalent in clinical terms and there are no specific tools to guide the clinician in choosing the proper LH activity preparation for OS. A recent analysis which appraised prospective and retrospective studies comparing $\mathrm{hMG}$ and $\mathrm{rFSH}+\mathrm{rLH}$ concluded that there is insufficient evidence to form any certain conclusions in favor of a particular source of preparation containing LH activity (14).

The current study was designed to compare OS outcome of two commercially available preparations with different source of LH bioactivity: rFSH + rLH in a fixed 2:1 ratio (Pergoveris ${ }^{\circledR}$, Merck, Darmstadt, Germany) and HP-hMG, containing urinary FSH and LH activity provided by hCG in a fixed 1:1 ratio (Menopur®, Ferring pharmaceuticals).

\section{Materials And Methods:}


We reviewed the computerized files of all consecutive women admitted to our IVF unit at the Sheba Medical Centre between March 2019 and March 2021. Inclusion criteria included patients which underwent two consecutive IVF cycles using the flexible multiple dose GnRH antagonist, where one OS included r-LH (Pergoveris) and the other included urinary hCG, HP-hMG, (Menopur).

OS was started on cycle day 2-3. OS included cycles started with rFSH preparation (Gonal F), which was converted to rFSH + rLH or HP-hMG from the day of GnRH antagonist administration (termed "COMBO" cycles) or cycles started with rFSH + rLH or HP-hMG from the beginning of stimulation (termed "ALL" cycles). The ovarian response was monitored by ultrasound and estradiol and progesterone levels from stimulation day 5 onwards. Once a leading follicle reached $13-14 \mathrm{~mm}$, and/or the estradiol levels exceeded $400 \mathrm{pg} / \mathrm{ml}$, co-treatment with $\mathrm{GnRH}$ antagonist was started. When the ovarian response was adequate, defined as at least three follicles measured above $17 \mathrm{~mm}$, a trigger for ovulation was administered using hCG, GnRH agonist or both. Routine IVF or intra-cytoplasmic sperm injection (ICSI) were performed as accepted.

The initial gonadotropin dosage, COMBO/ALL protocol and the medication for ovulation trigger were determined by the treating physician and according to the patients' clinical characteristics, ovarian reserve tests and the indication for fertility treatment. The elimination of bias in this selection, for the purposes of this study, was achieved by including only patients using the same initial FSH dosage, the same day of stimulation in which LH preparation was added, and the same ovulation trigger and mode of fertilization during the two consecutive treatment cycles. The time period between the two cycles was up to three months.

Data regarding patients' demographic and clinical characteristics and the response to OS and IVF treatment related variables was collected from the computerized clinical files. Primary outcome measure was the rate mature oocytes retrieved. Secondary outcome measures were the duration of stimulation, total dosage of gonadotropin used, number of mature and fertilized oocytes, number of top-quality embryos, defined as embryos with $\geq 7$ blastomeres and $<10 \%$ fragmentation on day-3 and pregnancy rate defined as positive beta-hCG test performed two weeks after an embryo transfer. To further examine the association between the LH type and ovarian stimulation characteristics, a subgroup analysis was carried out according to the OS protocol- "COMBO" and "All" cycles.

Statistical analysis was performed using the Statistical Package of Social Science version 27 (IBM Corp., USA). Categorial variables are presented as number of cases and percentage. Continuous data are presented as mean and standard deviation or median and inter-quartile range depending on normality test, as appropriate. Comparison of categorial variables were analysed by Mcnemar's test, and continuous variables by paired Students' t-test, as appropriate. A two tailed p-value of $<0.05$ was considered statistically significant.

Sample size calculation was based on a recent analysis comparing HMG and recombinant FSH plus LH during IVF cycles (14), using the rate of mature oocytes per cycle as the primary end point. Based on the expected difference of $7.2 \%$ between the rate of mature oocytes in the different treatment cycles, and a 
standard deviation of 19.9 in the rFSH + rLH treatment cycles and 16.2 in the HP-hMG treatment cycles, 53 patients were required to detect a significant difference with $80 \%$ power and $5 \%$ type 1 error.

The study was approved by the institutional review board of Sheba medical center (SMC-19-6610). All methods were performed in accordance with the relevant guidelines and regulations. Sheba Medical Center institutional review bord approved a waiver of informed consent, due to the retrospective nature of the study and the anonymous analysis of the clinical data.

\section{Results:}

The study included 53 patients which underwent two consecutive IVF cycles, each with a different LH activity preparation, one included rLH and the other HP-hMG. Demographic and basic clinical characteristics are presented in Table 1. 32 (60.4\%) patients had two "COMBO" cycles, in which OS started with $\mathrm{rFSH}$ only that was replaced by $\mathrm{rFSH}+\mathrm{rLH}$ or HP-hMG from day of GnRH antagonist treatment. The other 21 (39.6\%) patients had two "All" cycles, in which HP-hMG or rFSH + rLH were started from the beginning of OS. In 18 (35.8\%) patients, the first cycle included rFSH + rLH treatment, which was converted to HP-hMG in the second, consecutive cycle. 
Table 1

Basic characteristics of the study cohort and fertility treatment.

\begin{tabular}{|l|l|}
\hline & $\mathbf{n}=\mathbf{5 3}$ \\
\hline Age & $34.5 \pm 5.5$ \\
\hline BMI & $22.7 \pm 5.9$ \\
\hline FSH & $7.4(6,9)$ \\
\hline LSH to LH & $4.7(3,7)$ \\
\hline Infertility treatment indication & $1.7 \pm 0.8$ \\
\hline Fertility preservation & \\
\hline Poor ovarian reserve & $21(39.6)$ \\
\hline PGT & $3(5.6)$ \\
\hline Male factor & $14(26.4)$ \\
\hline Tubal factor & $7(13.2)$ \\
\hline Unexplained & $2(3.7)$ \\
\hline Other & $4(7.5)$ \\
\hline COMBO cycles & $2(3.7)$ \\
\hline Final follicular maturation & $32(60.4)$ \\
\hline hCG & \\
\hline GnRH agonist & $7(13.2)$ \\
\hline Dual trigger a & $32(60.3)$ \\
\hline Pergoveris first cycle & $14(26.4)$ \\
\hline Data are presented as mean \pm SD, median (intra-quartile range) or numbers and percentage. \\
\hline a-Dual trigger- hCG + GnRH agonist. & \\
\hline
\end{tabular}

The stimulation characteristics of the two cycles are presented in Table 2. There were no differences regarding the duration of stimulation or the total dose of $\mathrm{FSH}$ needed for stimulation between the two treatment cycles. As expected, the dose of $\mathrm{LH}$ in the treatment cycles including $\mathrm{rFSH}+\mathrm{rLH}$ was significantly lower compared with the HP-hMG treatment cycles, due to the contents of the medication which contains $\mathrm{rFSH}$ and $\mathrm{rLH}$ in a 2:1 ratio. 
Table 2

Ovarian stimulation outcome.

\begin{tabular}{|llll|}
\hline & MENOPUR & PERGOVERIS & P value \\
\hline Days of stimulation & $10.2 \pm 1.6$ & $10.4 \pm 1.4$ & 0.5 \\
\hline Total FSH & $3210 \pm 1484$ & $3169 \pm 1448$ & 0.7 \\
\hline Total LH & $2498 \pm 1617$ & $1243 \pm 775$ & $<0.01$ \\
\hline E2 level on trigger day (pmol/L) & $7191 \pm 4078$ & $8129 \pm 5330$ & 0.1 \\
\hline P level on trigger day (nmol/L) & $2.1 \pm 1$ & $2.5 \pm 1.4$ & 0.01 \\
\hline Endometrial thickness on trigger day (mm) & $9.7 \pm 2.8$ & $9.7 \pm 2.3$ & 0.4 \\
\hline Number of follicles $>13$ mm on trigger day & $9.3 \pm 4.4$ & $11.5 \pm 6.7$ & 0.03 \\
\hline Number of oocytes & $10.4 \pm 5.5$ & $11.5 \pm 6.1$ & 0.12 \\
\hline Number of M2 oocytes & $8.3 \pm 4.6$ & $10 \pm 5.8$ & 0.01 \\
\hline Rate of M2 oocytes & $0.8 \pm 0.2$ & $0.9 \pm 0.13$ & 0.07 \\
\hline Number of 2 PN & $6.4 \pm 3.6$ & $8.5 \pm 5.9$ & 0.05 \\
\hline Number of top-quality embryos (day 3) & $3.4 \pm 3$ & $4.0 \pm 3$ & 0.2 \\
\hline Rate of top-quality embryos (TOP/oocyte) & $0.3 \pm 0.2$ & $0.3 \pm 0.2$ & 0.9 \\
\hline Rate of top-quality embryos (TOP/2PN) & $0.5 \pm 0.3$ & $0.5 \pm 0.3$ & 0.7 \\
\hline Data are presented as mean \pm SD & & & \\
\hline PN- pronuclei & & & \\
\hline
\end{tabular}

Estradiol levels at the day of ovulation trigger were comparable between the two groups. Nonetheless, the progesterone levels at the day of stimulation trigger were significantly higher in the rFSH + rLH cycles compared with the HP-hMG cycles $(2.5 \pm 1.4 \mathrm{nmol} / \mathrm{L}$ vs $2.1 \pm 1 \mathrm{nmol} / \mathrm{L}$ respectively, $\mathrm{p}<0.01)$.

The rate of mature oocyte did not differ between the groups ( 0.9 in the rFSH + rLH cycles vs 0.8 in the HPhMG cycles, $p=0.0 .7)$. Nonetheless, the mean number of mature oocytes retrieved in the rFSH $+\mathrm{rLH}$ treatment cycles was significantly higher compared to the HP-hMG treatment cycles ( $10 \pm 5.8$ vs $8.3 \pm 4.6$, respectively, $P=0.01)$. Likewise, the mean number of fertilized oocytes was significantly higher in the rFSH + rLH cycles compared with the HP-hMG cycles ( $8.5 \pm 5.9$ vs $6.4 \pm 3.6$, respectively, $p=0.05)$. In a separate sub analysis of the "COMBO" cycles, these differences were most marked; the mean number of matured oocytes and the mean number of fertilized oocytes were 11 and 8.8 in the rFSH + rLH cycles, compared with 9 and 5.8 in the HP-hMG cycles ( $p=0.04$ and 0.03 respectively) (Table 3 ). 
Table 3

Sub analysis of COMBO cycles

\begin{tabular}{|llll|}
\hline & MENOPUR & PERGOVERIS & P value \\
\hline Days of stimulation & $10 \pm 1.6$ & $10.4 \pm 1.3$ & 0.2 \\
\hline Total FSH & $2939 \pm 1484$ & $2895 \pm 1321$ & 0.4 \\
\hline Total LH & $1763 \pm 1201$ & $926 \pm 580$ & $<0.01$ \\
\hline E2 level on trigger day (pmol/L) & $7460 \pm 4227$ & $8094 \pm 5590$ & 0.3 \\
\hline P level on trigger day (nmol/L) & $2.1 \pm 1.1$ & $2.4 \pm 1.1$ & 0.05 \\
\hline Endometrial thickness on trigger day (mm) & $9.4 \pm 1.9$ & $9.8 \pm 2.2$ & 0.2 \\
\hline Number of follicles > 13 mm on trigger day & $10.4 \pm 5$ & $11.5 \pm 6.2$ & 0.2 \\
\hline Number of oocytes & $10.9 \pm 5.3$ & $12.6 \pm 6.3$ & 0.09 \\
\hline Number of M2 oocytes & $9.0 \pm 5.4$ & $11 \pm 6$ & 0.04 \\
\hline rate of M2 oocytes & $0.8 \pm 0.2$ & $0.9 \pm 0.1$ & 0.6 \\
\hline number of 2 PN & $5.8 \pm 3.8$ & $8.8 \pm 6.1$ & 0.03 \\
\hline number of top-quality embryos (day 3) & $2.9 \pm 3.1$ & $4.3 \pm 3.5$ & 0.11 \\
\hline Rate of top-quality embryos (TOP/oocyte) & $0.2 \pm 0.1$ & $0.3 \pm 0.2$ & 0.17 \\
\hline Rate of top-quality embryos (TOP/2PN) & $0.4 \pm 0.2$ & $0.5 \pm 0.3$ & 0.4 \\
\hline Data are presented as mean \pm SD & & & \\
\hline
\end{tabular}

There was no difference between the treatment cycles regarding the number of top-quality embryos nor the ratio of top-quality embryos to the number of oocytes retrieved or fertilized oocytes (Table 2). The pregnancy rate per fresh transfer was higher among the HP-hMG treatment cycles $(4 / 14,29 \%)$ compared with the $\mathrm{rFSH}+\mathrm{rLH}$ cycles $(2 / 14,15 \%)$, although this difference did not reach statistical significance $(\mathrm{p}=$ $0.3)$.

\section{Discussion:}

In the present study, we compared the OS outcomes of two LH activity preparations- recombinant $\mathrm{LH}$ and $\mathrm{HP}-\mathrm{hMG}$, in which the LH activity is derived mostly from hCG. Patients treated with $\mathrm{rFSH}+\mathrm{rLH}$ yielded significantly higher numbers of mature oocytes and fertilized oocytes, with non-significantly lower pregnancy rate per transfer ( $15 \%$ vs $29 \%$, respectively. $P=0.3)$ compared to those treated with HP-hMG.

LH and hCG are heterodimeric glycoprotein hormones sharing approximately $85 \%$ structural identity. Both of them bind to a mutual LH/choriogonadotropin receptor (LH/CGR), a G protein-coupled receptor with an 
extra-cellular binding domain to which LH and hCG bind in distinct, specific regions. The distinct molecular structure of the beta-LH and beta-hCG subunits results in different conformational changes of the LH/CGR, leading to activation of different intracellular cascades (5). The post receptor, intra-cellular reaction, includes an activation of adenylate cyclase, which increases the intracellular pool of cAMP and results in steroidogenesis, and an extracellular signal-regulated protein kinases 1 and 2 (ERK1/2) and AKT, with putative roles in cell proliferation, differentiation and survival (7). In vitro studies found that hCG is five times more potent than LH with respect of CAMP production and steroidogenesis pathway, while LH strongly activates ERK1/2 and AKT pathways, thus induces proliferation and anti-apoptotic effect $(6,15)$. Although in vitro models provided the evidence of hormone- specific actions, whether they influence differently on in vivo OS response remains unclear and published evidence of the difference between $\mathrm{rLH}$ and $\mathrm{hCG}$ during OS is surprisingly scarce.

The main findings of our study were higher number of mature oocytes retrieved and higher number of oocytes fertilized in the rFSH + rLH compared with the HP-hMG treatment cycles. These differences confirm that rLH and hCG poses different influences also in vivo during OS cycles. The higher number of mature oocytes may be due to the synergistic effect of FSH and LH on follicular growth, proliferation and maturation. Moreover, the addition of LH shifts granulosa cells from proapoptotic to proliferative pathways, in contrast to hCG which has proapoptotic effect mediated by relatively higher intracellular cAMP concentrations $(16,17)$. Gomez-Palomares et al evaluated the OS outcomes in women over 38 years old supplemented with rFSH + rLH or hMG(18). Similar to our results, they found a higher rate of mature oocytes in the group treated with $\mathrm{rFSH}+\mathrm{rLH}$. Pacchiarotti et al in their randomized controlled trial comparing $\mathrm{rFSH}+\mathrm{rLH}$ with $\mathrm{hMG}$, also found higher number of oocytes retrieved and higher number of mature oocytes in the rFSH + rLH cycles, although the rate of matured oocytes per overall oocytes yield was higher in the hMG cycles (19). Unlike our study that included down regulation by GnRH antagonist, Pacchiarotti et al used the long GnRH agonist down regulation protocol, which may influence differently the initial follicular reaction to rLH or hCG.

When comparing the IVF treatment outcomes of $\mathrm{rFSH}+\mathrm{rLH}$ to HP-hMG, we must also consider the differences in the type of FSH molecules, which may be related to the variation observed between the two treatments. The higher mature oocytes yield demonstrated in the rFSH + rLH treatment cycles, may be derived from the greater effectiveness of the rFSH isoform compared with urinary FSH, rather than from the effect induced by rLH, as it is well-established that $\mathrm{rFSH}$ leads to higher follicular recruitment compared with HP-hMG $(1,8,20)$.

Another interesting finding of our study was higher progesterone levels in the rFSH + rLH cycles compared with the HP-hMG cycles. This finding might be explained by the higher number of follicles in the rFSH + rLH treatment cycles. However, we must also consider the different effect of rLH and hCG on the steroidogenesis process as an alternative explanation for the different endocrine profile. Previous studies comparing progesterone levels following OS with rFSH or HP-hMG have revealed similar findings; rFSH stimulation alone was associated with higher progesterone levels at the end of stimulation, even after adjusting for ovarian response $(1,8,21,22)$. In accordance to our study, a study by Sebag-Peyrelecade et 
al which compared the progesterone levels of $\mathrm{rFSH}+\mathrm{rLH}$ treatment with those of hMG treatment, revealed that supplementation of rLH was not sufficient to decrease the progesterone levels to those observed in the hMG treatment cycles, unrelated to the degree of ovarian response(21). Therefore, we may deduce that the lower progesterone levels measured in the hMG cycles are attributed to the hCG content.

In the present study, we did not find any differences regarding the rate of mature oocytes nor the number of top-quality embryos between the rFSH + rLH and HP-hMG treatments, despite of the initial higher number of fertilized oocytes in the rFSH $+\mathrm{LH}$ treatment cycles. This observation suggests that although $\mathrm{rFSH}+\mathrm{rLH}$ is more potent in follicular genesis and oocytes yield, HP-hMG may favor embryonal maturation. In accordance with our findings, previous studies that compared the use ofHP-hMG vs rFSH, such as the MERiT and the MEGASET trials, have found that HP-hMG results in higher proportion of topquality embryos $(8,20)$.

Of not, although not statistically significant, the pregnancy rate per fresh transfer was higher among the HP-hMG treatment cycles compared to the $\mathrm{rFSH}+\mathrm{rLH}$ cycles. This difference in pregnancy rate may be a consequence of the higher progesterone levels during the rFSH + rLH cycles, which negatively influence the endometrial receptivity and the essential synchronization between the embryo and the endometrium. Nonetheless, because the present study is not sufficiently powered to detect differences in pregnancy rate, this data should be viewed with caution.

A major strength of our study is that we compared the different LH activity preparations in the same cohort of patients. The fact that all women that participated in our study had two consecutive treatment cycles using rFSH + rLH in one cycle and hMG in the other, helps to eliminate multiple bias factors and to attribute the study results to the different treatment preparations.

A limitation of our study is the lack of data regarding clinical pregnancy or live birth rates. Nonetheless, one may say that when the question of investigation is the effect of LH activity products on OS, the primary outcome has to be the first measurable parameter of gonadotropin influence, i.e., the ovarian response. Moreover, the majority of our study cohort comprised of patients which underwent IVF treatment for social fertility preservation. In this section of patients, we are most interested in achieving an optimal number of mature oocytes.

In conclusion, our study suggests that gonadotropins preparations have different influence on OS outcome, proving the necessity of tailoring a specific gonadotropin regimen when assembling a treatment protocol. $\mathrm{rFSH}+\mathrm{rLH}$ resulted in higher number of matured oocytes and fertilized oocytes, while the lack of difference regarding the number of top-quality embryos between the preparations might suggest an encouraging effect of hMG on oocytes and embryo quality. These treatment characteristics, derived by different LH preparation, reflect the physiological role of these molecules as previously indicated by in vitro data.

\section{Declarations:}




\section{Ethic approval and consent to participate:}

The study was approved by the institutional review board of Sheba medical center (SMC-19-6610). All methods were performed in accordance with the relevant guidelines and regulations. Sheba Medical Center institutional review bord approved a waiver of informed consent, due to the retrospective nature of the study and the anonymous analysis of the clinical data.

\section{Consent of publication:}

Not applicable.

\section{Availability of data and material:}

All authors have a secured access to the study data. Data are available from the corresponding author on request.

\section{Funding:}

The authors have no funding resource to declare.

\section{Conflict of interest:}

The authors have no conflict of interest to declare.

\section{Authors' contribution:}

M. K.- Study design, data collection, data analysis and interpretation of data. manuscript writing.

O. G.- Study design, data collection, revising manuscript.

J. H.- Conception and design of study, revising manuscript.

R. N.- Conception and design of study, revising manuscript.

E. Z.- Conception and design of study, revising manuscript.

O. L.- Conception and design of study, revising manuscript.

R. O.- Substantial contributions to the conception or design of the work and the acquisition, analysis, or interpretation of data, revising manuscript.

\section{References:}

1. Bosch E, Labarta E, Kolibianakis E, Rosen M, Meldrum D. Regimen of ovarian stimulation affects oocyte and therefore embryo quality. Fertility and Sterility. 2016;105(3):560-570. 
2. Levy DP, Navarro JM, Schattman GL, Davis OK, Rosenwaks Z. The role of LH in ovarian stimulation: Exogenous LH: Let's design the future. Human Reproduction. 2000;15(11):2258-65.

3. Lunenfeld B, Bilger W, Longobardi S, Alam V, D'Hooghe T, Sunkara SK. The development of gonadotropins for clinical use in the treatment of infertility. Frontiers in Endocrinology. 2019;10:429.

4. The Practice Committee of the American Society for Reproductive Medicine, Birmingham A. Gonadotropin preparations: past, present, and future perspectives. Fertil Steril. 2008; 90(5 Suppl):S13-20.

5. Casarini L, Santi D, Brigante G, Simoni M. Two hormones for one receptor: Evolution, biochemistry, actions, and pathophysiology of LH and hCG. Endocrine Reviews. 2018;39(5):549-592.

6. Casarini L, Lispi M, Longobardi S, Milosa F, la Marca A, Tagliasacchi D, et al. LH and hCG Action on the Same Receptor Results in Quantitatively and Qualitatively Different Intracellular Signalling. PLoS One. 2012;7(10):e46682.

7. Smitz J, Platteau P. Influence of human chorionic gonadotrophin during ovarian stimulation: An overview. Reproductive Biology and Endocrinology. 2020;18(1):80.

8. Andersen AN, Devroey P, Arce JC. Clinical outcome following stimulation with highly purified hMG or recombinant FSH in patients undergoing IVF: A randomized assessor-blind controlled trial. Hum Reprod. 2006; 21(12):3217-27.

9. Coomarasamy A, Afnan M, Cheema D, Van Der Veen F, Bossuyt PMM, Van Wely M. Urinary hMG versus recombinant FSH for controlled ovarian hyperstimulation following an agonist long downregulation protocol in IVF or ICSI treatment: A systematic review and meta-analysis. Hum Reprod. $2008 ; ; 23(2): 310-5$.

10. Al-Inany HG, Abou-Setta AM, Aboulghar MA, Mansour RT, Serour GI. Efficacy and safety of human menopausal gonadotrophins versus recombinant FSH: A meta-analysis. Reprod Biomed Online. 2008; 16(1):81-8.

11. Alviggi C, Conforti A, Esteves SC, Andersen CY, Bosch E, Bühler K, et al. Recombinant luteinizing hormone supplementation in assisted reproductive technology: a systematic review. Fertil Steril. 2018; 109(4):644-664.

12. Filicori M, Fazleabas AT, Huhtaniemi I, Licht P, Rao C V., Tesarik J, et al. Novel concepts of human chorionic gonadotropin: Reproductive system interactions and potential in the management of infertility. Fertility and Sterility. 2005;84(2):275-84.

13. Ziebe S, Lundin K, Janssens R, Helmgaard L, Arce JC. Influence of ovarian stimulation with HP-hMG or recombinant FSH on embryo quality parameters in patients undergoing IVF. Hum Reprod. 2007; 22(9):2404-13.

14. Orvieto R. HMG versus recombinant FSH plus recombinant LH in ovarian stimulation for IVF: does the source of LH preparation matter? Reproductive BioMedicine Online. 2019;39(6):1001-1006.

15. Choi J, Smitz J. Luteinizing hormone and human chorionic gonadotropin: Origins of difference. Molecular and Cellular Endocrinology. 2014; 383(1-2). 
16. Casarini L, Reiter E, Simoni M. $\beta$-arrestins regulate gonadotropin receptor-mediated cell proliferation and apoptosis by controlling different FSHR or LHCGR intracellular signaling in the hGL5 cell line. Mol Cell Endocrinol. 2016;437:11-21.

17. Casarini L, Riccetti L, De Pascali F, Nicoli A, Tagliavini S, Trenti T, et al. Follicle-stimulating hormone potentiates the steroidogenic activity of chorionic gonadotropin and the anti-apoptotic activity of luteinizing hormone in human granulosa-lutein cells in vitro. Mol Cell Endocrinol. 2016; 422:103-114.

18. Gómez-Palomares J, Acevedo-Martín B, Andrés L, Ricciarelli E, Hernández E. LH improves early follicular recruitment in women over 38 years old. Reprod Biomed Online. 2006; 11(4):409-14.

19. Pacchiarotti A, Sbracia M, Frega A, Selman H, Rinaldi L, Pacchiarotti A. Urinary hMG (Meropur) versus recombinant FSH plus recombinant LH (Pergoveris) in IVF: A multicenter, prospective, randomized controlled trial. Fertil Steril. 2010;94(6):2467-9.

20. Witz CA, Doody K, Park J, Seifu Y, O’Brien K, Yankov V, et al. Highly purified human menotropin (HP$H M G$ ) versus recombinant follicle stimulating hormone (RFSH) in high responders undergoing in vitro fertilization (IVF): MEGASET-HR trial outcomes. Fertil Steril. 2017; 108(3)Suppl:e21-22.

21. Sebag-Peyrelevade S, El Hachem H, Gallot V, Genro VK, Fanchin R. The influence of exogenous $\mathrm{LH} / \mathrm{hCG}$ activity on serum progesterone levels on the day of hCG administration in in vitro fertilization. J Gynecol Obstet Biol Reprod (Paris). 2015; 44(6):524-31.

22. Sharara FI, Foster ED, Sinha A, Daftary GS, Heiser PW. Gonadotropin-specific follicular steroidogenesis in ovarian stimulation: evidence from the menopur in $\mathrm{GnRH}$ antagonist single embryo transfer - high responder (MEGASET-HR) trial. Fertil Steril. 2019; 112(3):e56. 